\title{
A comparative analysis of Caenorhabditis and Drosophila transcriptional changes in response to pathogen infection
}

Robert L. Unckless, Patrick A. Lansdon and Brian D. Ackley

Department of Molecular Biosciences, University of Kansas, Lawrence, KS, 66045

Running title: Immune divergence in invertebrates

Keywords: Innate immunity, RNA Sequencing, Transcriptomics, Pathogenic Bacteria

Corresponding Author:

Robert L. Unckless

1200 Sunnyside Ave

Lawrence, KS 66045

unckless@ku.edu

4 Figures, 1 Tables, 1 Supplemental Figure, 7 Supplemental Tables

31 Pages

Summary: 114 Words

Abstract: 264 Words

Introduction: 520 Words

Results: 1486 Words

Discussion: 628 Words

1) P a g e 
Immune divergence in invertebrates

\section{Article Summary}

We analyzed transcriptomic data from $C$. elegans and D. melanogaster to compare the expression of orthologous pairs of genes in response to bacterial pathogens. Our results indicated that only a handful of genes that are orthologous between species are differentially expressed in response to pathogens, but that the pattern of expression was different when comparing one-to-one orthologs versus those that are restricted to one of the two organisms. These results suggest that, although broad patterns of susceptibility to bacterial pathogens are conserved, the regulatory framework by which the organisms fight pathogens is less well conserved. Further our results suggest a more complete analysis of the evolutionary changes in organismal responses to pathogens is required. 
Immune divergence in invertebrates

\section{Abstract}

Drosophila melanogaster and Caenorhabditis elegans are well-used invertebrate models for studying the innate immune system. The organisms are susceptible to bacterial pathogens that include Pseudomonas species, (entomophilia - Drosophila) or (aeruginosa - Caenorhabditis), E. faecalis and $P$. rettgeri, which are or are related to human pathogens. Further, the consequences of exposure to these pathogens, in terms of organismal survival, are roughly equivalent when compared. That is, worms and flies are more susceptible to infection by Pseudomonas than E. faecalis, whereas organismal survival on E. faecalis and P. rettgeri are roughly the same in both. To better understand how these organisms are coordinating their responses to these bacterial pathogens we examined transcriptomes in infected animals. We grouped our analysis based on protein orthology. Of the 3611 pairs analyzed, we found genes whose responses were conserved across the different species at a higher than expected rate for two of the three pathogens. Interestingly within the animals, genes with 1:1 orthologs between species behaved differently. Such genes were more likely to be expressed in $D$. melanogaster, and less likely to be expressed in C. elegans. From this analysis we found that the gene nucleobindin (nucb-1/NUCB1 in C. elegans and D. melanogaster, respectively) was upregulated in both species in response to Gram negative bacteria. We used RNAi to knock down nucb-1 and found the treated animals were more susceptible to infection by the Gram negative pathogen P. rettgeri than controls. These results provide insight into some of the conserved mechanisms of pathogen defense, but also suggest that these divergent organisms have evolved specific means to orchestrate the defense against pathogens. 
Immune divergence in invertebrates

\section{Introduction}

The innate immune system is ancient and evolutionarily conserved across the animal kingdom. By contrast the adaptive immune system evolved in bony fish about 500 million years ago (BOEHM AND SWANN 2014). The function of the innate immune system is to protect organisms against pathogens such as bacteria, fungi and viruses. The broad repertoire and rapid evolution of pathogens appears to have had a compensatory effect in the adaptation of the innate immune system in animals. From this, there has been a general observation that immune genes are often amongst the fastest evolving genes in genomes (NIELSEN et al. 2005; SACKTON et al. 2007; Pujol et al. 2008; DoWNing et al. 2009; ObBARD et al. 2009; VILJAKAINEN et al. 2009; SHULTZ AND SACKTON 2019).

However, more recent work has suggested that evolution in the innate immune system is more nuanced than that. The innate immune system can be roughly divided into pathways that respond to bacterial pathogens (with differences in Gram-positive vs. Gram-negative), fungal pathogens and viruses. When examined within the different pathways, there is evidence for different signatures of adaptation between Drosophila species. Rapid evolution in immune genes seems to be specifically to the viruses they face (DUXBURY et al. 2019; HILL et al. 2019) or in response to other ecological pressures (HANSON et al. 2019). The evolution of immune genes in Caenorhabditis species is less studied and recurrent positive selection appears to be much less pronounced (PUJOL et al. 2008; SchULENBURG et al. 2008; DieRKING et al. 2016).

Caenorhabditis elegans (roundworms) and Drosophila melanogaster (fruit flies) have been important models for identifying fundamental mechanisms of how organisms 
fight infection(BUCHON et al. 2014; ERMOLAEVA AND SCHUMACHER 2014). Work from these systems have uncovered genes and pathways, many that are conserved in humans, that facilitate response to infection. Primarily, these include the Toll and Imd pathways (flies) and insulin/FOXO and MAP Kinase pathways (worms). However, the Toll and Imd pathways, while well-conserved from flies to humans, are largely absent in nematodes (SCHULENBURG et al. 2008). In flies the insulin/FOXO pathway is important, but less critical to the overall response.

However, reviewing the work done suggests that there has been more limited comparison of information obtained from these two systems. Interestingly, where it has been measured, pathogens exhibit roughly similar patterns of virulence in these organisms. For example, Pseudomonas species are more virulent than Enterococcus or Providencia, whereas Enterococcus and Providencia are roughly equivalent (SIM AND Hibberd 2016; MARtin et al. 2017; ARCHeR ANd Phillips 2018; TROHA et al. 2018; VASQUEZ-RIFO et al. 2019), B.D. Ackley, Personal Observation). This suggests that, even if the animals have invested different genetic resources in fighting these pathogens, the outcomes have not significantly improved. We find that genes differentially induced after exposure to Gram-positive and Gram-negative microbes are more likely to be lineage restricted in Drosophila but one-to-one orthologs (with Drosophila) in Caenorhabditis. Furthermore, there is weak, but significant correlation between fold change (between Gram-negative to Gram-positive exposure) between flies and worms. One particular gene, Nucb-1, was significantly biased toward expression after Gram-negative infection, and appears to play a role specifically in fighting Gram-negative infections. 


\section{Methods}

Data acquisition: Drosophila melanogaster

All D. melanogaster sequencing data was published previously (TROHA et al. 2018). We downloaded data from the sequence read archive (SRA) for 12 hours post infection with Enterococcus faecalis, Escherichia coli, Providencia rettgeri and Pseudomonas entomophila. Details for all sequencing data sets is presented in Table S1.

Data acquisition: Caenorhabditis elegans

C. elegans (N2, var. Bristol) were reared on Escherichia coli (OP50) on nematode growth media (NGM) plates using standard conditions (BRENNER 1974). For pathogen exposure, NGM plates were seeded with $250 \mu \mathrm{L}$ of bacteria, Pseudomonas aeruginosa (PA14), Enterococcus faecalis (OG1RF), Providencia rettgeri (D. mel isolate) or OP50 (control), and incubated overnight at $37^{\circ} \mathrm{C}$. Approximately $2,000 \mathrm{~L} 4$ stage worms were transferred to prepared NGM plates and incubated at $20^{\circ} \mathrm{C}$ for 24 hours. After 24 hours, worms were washed with M9 buffer three times to remove any residual bacteria. The worms were resuspended in $100 \mu \mathrm{L}$ of M9 buffer and mechanically disrupted in liquid nitrogen using a ceramic mortar and pestle. Frozen tissue was transferred to a microcentrifuge tube and $1 \mathrm{~mL} T R I Z O L$ reagent was added. The tubes were flash frozen in liquid nitrogen and stored at $-80^{\circ} \mathrm{C}$. Three biological replicates were collected for each worm and pathogen strain. 
Frozen worm tissue was thawed, vortexed for 5 seconds and incubated at room temperature for 5 minutes. After the addition of $470 \mu \mathrm{L}$ chloroform (mixed by inversion and phase separated for 2 minutes at room temperature), the samples were centrifuged at 15,000 RPM at $4^{\circ} \mathrm{C}$ for 15 minutes. The upper aqueous phase containing RNA (approximately $600 \mu \mathrm{L}$ ) was transferred to a new RNase-free Eppendorf tube. Total RNA extraction was carried out using the Monarch RNA Cleanup Kit (New England Biolabs) according to the manufacturer's instructions. Total RNA was quantified using the Qubit (Thermofisher Scientific) and RNA quality and integrity was assessed using the Agilent Tapestation 2200 (Agilent Technologies). Sequence libraries were prepared using the TruSeq Stranded mRNA Library Prep Kit (Illumina) and sequenced using single-end $1 \times 75$ bp sequencing on the Illumina NextSeq 550 platform.

Gene expression quantification:

Raw reads were mapped to indexed transcriptomes using Kallisto version 0.46.0 (BRAY et al. 2016) then total counts were rounded to the nearest integer and imported into $R$ version 5.1.3 (R DeVELOPMENT CORE TEAM 2015) for analysis. We used all mRNA transcripts from D. melanogaster FlyBase release r6.30 (THURMOND et al. 2019) and the longest transcripts from C. elegans WormBase release WBcel235 (HARRIS et al. 2020) as references. We assessed differential expression of transcripts using DESeq2 (LOVE et al. 2014).

Creation of ortholog lists: 
We obtained a list of orthologs shared between $D$. melanogaster and C. elegans from InParanoid8 (SONNHAMMER AND OSTLUND 2015). Briefly, we navigated to the "Browse the Database" page, selected Drosophila melanogaster and Caenorhabditis elegans as the two species and employed a custom script to both parse the data and distinguish between one-to-one orthologs and one-to-many orthologs.

Experimental infections in Drosophila:

To determine the role of NUCB1 in immune defense, we crossed a fat body / hemocyte driver (C564 - Bloomington Drosophila Stock Center \#6982) to either a NUCB1 RNAi construct (Bloomington Drosophila Stock Center \#44019) or empty cassette (Bloomington Drosophila Stock Center \#36304). All flies were maintained on a standard molasses diet at $23^{\circ} \mathrm{C}$ with a 24-hour light cycle (12 hours light followed by 12 hours dark). Adults were transferred to new food vials after eclosion and males were infected at three to six days old. All infections took place in the morning (between 1 and 2 hours after "daylight"). Infections were performed by septic injury using a minutien pin dipped in either sterile Luria Bertani media (control), P. rettgeri (diluted to $\mathrm{OD}_{600}=1.00$ \pm 0.02 ), or $E$. faecalis (diluted to $\mathrm{OD}_{600}=1.5 \pm 0.02$ ). For survival assays, the number of flies alive was noted every morning for seven days. For bacterial load, we homogenized groups of three flies in 1X PBS, diluted 1:20, then plated the dilute homogenate on LB agar using the WASP Touch spiral plater (Don Whitley Scientific, West Yorkshire, UK). These plates were incubated at $37^{\circ} \mathrm{C}$ for about 18 hours then counted using a Flash \& Go Plate Counter (Neu-tec Group Inc., Farmingdale, NY, USA). 
Experimental infection in C. elegans:

Wild-type (N2) animals were reared on nucb-1 RNAi-expressing bacteria (Source Bioscience, Nottingham, UK). nucb-1 RNAi bacteria were grown overnight at $37^{\circ} \mathrm{C}$ in $1.5 \mathrm{~mL}$ LB plus ampicillin, tetracycline and $1 \mathrm{mM}$ IPTG with shaking. $0.2 \mathrm{ml}$ of the overnight cultures were aliquoted onto NGM plates containing carbenicillin, tetracycline, and $1 \mathrm{mM} \mathrm{IPTG}$ and grown overnight at $37^{\circ} \mathrm{C}$. Healthy $\mathrm{L} 4$ animals were transferred to the nucb-1 RNAi plates or OP50 (E. coli) control plates and allowed to produce offspring. Offspring were transferred to freshly prepared plates and reared for 2 days. 20-30 L4 animals from the RNAi or control plates were transferred to NGM plates seeded with E. coli, E. faecalis or P. rettgeri and then maintained at $20^{\circ} \mathrm{C}$ for the remainder of the experiment. Every 24 hours living animals were transferred to NGM plates with the same bacterial species and the number of living and dead animals was counted. Animals were transferred to fresh plates daily, until all animals were dead. RNAi was done in triplicate.

\section{Results}

One-to-one orthologs are less likely to be differentially expressed than other genes in worms but not flies.

We measured gene expression after four different treatments in both Drosophila and Caenorhabditis. Principal component analysis of gene expression reveals that in both 
flies and worms, the sole Gram-positive pathogen, a moderately virulent strain of Enterococcus faecalis, clustered distinctly from other samples (Figure S1). In Drosophila, the two pathogenic Gram-negative microbes (Pseudomonas entomophila and Providencia rettgeri) clustered together while the non-pathogenic Escherichia coli) was somewhat intermediate between E. faecalis and the pathogenic Gram-negative pathogens. In Caenorhabditis, E. coli is the food source so we would expect very little immune response. Strangely, however, E. coli clusters with the relatively virulent $P$. rettgeri while the very virulent Pseudomonas aeruginosa is quite different. Thus, it appears that both broad bacterial taxonomy and virulence drive expression similarities in both flies and worms, but are not the only factors involved.

Since two of the most highly immune-induced pathways in Drosophila (Imd and Toll) do not exist in Caenorhabditis, we hypothesized that immune induction would tend to involve lineage-specific genes and not conserved orthologs. We therefore assigned all genes in each taxa as lineage restricted, one-to-many orthologs or one-to-one orthologs (with orthology considered compared to the other taxa: flies to worms and worms to flies). This resulted in 3543/3580 one-to-one orthologs, 3428/3298 one-to-many orthologs and 10854/9102 lineage restricted genes in Drosophila and Caenorhabditis, respectively. In flies, there were 79,331 and 248 differentially expressed genes comparing $E$. faecalis to $E$. coli, $P$. aeruginosa and $P$. rettgeri, respectively (FDR=0.05). In worms, there were 5867,6375 and 5868 differentially expressed genes comparing $E$. faecalis to E. coli, $P$. aeruginosa and $P$. rettgeri, respectively (FDR=0.05). In Drosophila, lineage restricted genes were most likely to be differentially expressed, followed by one10 | P a g e 
to-many orthologs, then one-to-one orthologs when comparing infection with $E$. faecalis to any of the Gram-negative bacteria (Figure 1A). In all cases, orthology category was significantly associated with the likelihood of being differentially expressed, however, Tukey honestly significant difference tests were significant for only some pairwise comparisons (Supplemental Table 2). The pattern for Caenorhabditis, however, is quite different: lineage restricted genes are least likely to be differentially expressed, followed by one-to-many orthologs and one-to-one orthologs are most differentially expressed (Figure 1B, Supplemental Table 2).

Expression differences between individuals exposed to Gram+ and Gram-pathogens are weakly correlated in one-to-one orthologs in divergent hosts.

When considering only one-to-one orthologs, the $\log _{2}$ fold change after exposure to E. faecalis vs. all three Gram-negative pathogens was positively correlated, but weakly. The correlation for $E$. coli was weakest $(\rho=0.042, \mathrm{P}=0.086$ ), with both Pseudomonas $(\rho=0.172, \mathrm{P}<0.0001)$ and $\mathrm{P}$. rettgeri $(\rho=0.170, \mathrm{P}<0.0001)$ similar to each other, but only moderately correlated. This suggests that while there is some degree of conservation of the overall difference in expression response to Gram-positive vs. Gram-negative pathogens, the pattern is relatively weak. A handful of genes were significantly differentially expressed (in the same direction) in both flies and worms when comparing exposure to E. faecalis to Pseudomonas or Providencia (Table 1). 
Genes differentially expressed comparing infection with Pseudomonas to E. faecalis: CG14210/Y40B1B.7 was Gram-positive biased in both animals but is poorly characterized and is an ortholog of the human CCDC86 gene (coiled-coil domain containing 86) which localizes to the nucleolus (THURMOND et al. 2019; HARRIS et al. 2020). NUCB1/nucb-1 was Gram-negative biased in both animals and is a nucleobindin protein involved in defense against Gram-negative bacteria in Drosophila (BERKEY et al. 2009) and is expressed in the intestines of Caenorhabditis (HARRIS et al. 2020). Galt/ZK1058.3 was Gram-positive biased in both animals is a galactose-1-phosphate uridylyltransferase associated with galactose homeostasis in Drosophila and without phenotypic data in Caenorhabditis (THURMOND et al. 2019). In addition, Galt was upregulated in Drosophila after infection with Octosporea (a microsporidian that normally infects Daphnia) (ROXSTROM-LINDQUIST et al. 2004). Esyt2/esyt-2 was Gramnegative biased and is a synaptotagmin-like protein involved in lipid transport, among other things (THURMOND et al. 2019; HARRIS et al. 2020).

Genes differentially expressed comparing infection with P. rettgeri to E. faecalis: ade2/pfas-1 was significantly Gram-positive biased after exposure and is a phosphoribosylformylglycinamidine synthase and is involved in purine synthesis and is expressed in the fat body in Drosophila and the intestines in Caenorhabditis (THURMOND et al. 2019; HARRIS et al. 2020). CG6218/WO6B4.2 was gram-negative biased in both animals and is a $\mathrm{N}$-acetylglucosamine kinase involved in carbohydrate phosphorylation and influences dauer lifespan in Caenorhabditis (XIE AND RoY 2012). 
GO Analysis reveals different pathways influenced by infection

Several Gene Ontology categories were enriched when comparing exposure to the Gram-positive E. faecalis to the three Gram-negative microbes (Supplemental Table 3). Focusing on molecular function, the many categories enriched for one pathogen were also enriched for other categories in both Drosophila (Figure 3A) and Caenorhabditis (Figure 3B), with the important caveat that in all cases the three different Gram-negative microbe treatments are being compared to the same Gram-positive E. faecalis treatment. Overlaps are significantly more common than expected by chance in both species in all pairwise comparisons (Supplemental Table 4). However, the only molecular function Gene Ontology category shared between Drosophila and Caenorhabditis after exposure to any of the microbes was "GO:0001055 - RNA Polymerase II Activity".

Oral infection in Drosophila is largely consistent/inconsistent with previous results

Since the above analysis compares systemic infection in Drosophila to oral infection in Caenorhabditis, we also compared Drosophila gene expression 16 hours after oral infection with P. entomophila (BOU SLEIMAN et al. 2020). However, in this analysis guts, not whole animals were used as a source of RNA. Also, in this case, we compared control expression (standard diet) to expression 16 hours after exposure to $P$. entomophila in Drosophila. The best contrast in Caenorhabditis was comparing exposure to $E$. coli (which is the standard worm diet) to exposure to $P$. aeruginosa. So 13 | P a g e 
in this section, for both taxa, we are comparing standard diet to a highly virulent oral Pseudomonas infection. Like Drosophila systemic infection, lineage restricted genes (21.1\%) were significantly more likely to be differentially expressed than one-to-one orthologs $(16.9 \%)$, but one-to-many orthologs were actually the most likely class to be differentially expressed $(23.7 \%$, Table S2). In this new comparison (Pseudomonas vs. Escherichia) in Caenorhabditis, the patterns of differential expression and orthology look very much like Pseudomonas vs. E. faecalis, suggesting that the patterns of differential expression might be driven by the virulence of Pseudomonas.

The $\log _{2}$ fold change of one-to-one orthologs after oral infection was negatively correlated between Drosophila and Caenorhabditis (rho=-0.087, $\mathrm{P}<0.0001$ ), suggesting that perhaps the whole-animal vs. gut-only approach led to this negative correlation. A handful of genes were significantly induced in both animals and include $c v-2 / T 01 D 3.6$, Jra/jun-1, vri/atf-2, alpha-Man-Ilb/aman-3, CG10681/kxd-1, spds/spds-1, CG10184/R102.4, and CG32549/Y71H10B.1 (fly gene symbol/worm gene symbol). Jra/jun-1 is of particular interest because it is a negative regulator of NF-KappaB mediated activation of antimicrobial peptides in Drosophila and mutant heterozygotes had reduced survival after infection with generally avirulent $E$. coli (KIM et al. 2007). Interestingly, jun-1 mutants in $C$. elegans had increased survival after infection with $P$. aeruginosa (KAO et al. 2011). alpha-Man-Ilb/aman-3 has alpha-mannosidase activity and is associated with the encapsulation response in Drosophila (MORTIMER et al. 2012).

No gene ontology terms were significantly overrepresented in both Drosophila and Caenorhabditis (Supplementary Table 4). 
Experimental infections reveal a Gram-negative specific role in immune defense for Nucb-1

The nucb-1 / NUCB1 Orthologs were significantly Gram-negative biased in both flies and worms. This is interesting since the Toll and IMD pathways that regulate defense against Gram-positive and Gram-negative microbes in D. melanogaster are missing in C. elegans. We therefore tested whether knocking down nucb-1 would show an immune phenotype after challenge with $P$. rettgeri or E. faecalis. First, there was a significant interaction between treatment and genotype on log-transformed total bacteria per fly (Figure 4A, Supplementary Table 5, $\mathrm{P}=0.031$ ) 24 hours after infection. The mean $E$. faecalis load for males with NUCB1 knocked down was $8 \%$ of controls $\left(\bar{x}_{\text {Control }}=\right.$ $\left.3560.5, \bar{x}_{N U C B 1}=294.1\right)$. However, the mean P. rettgeri load for males with NUCB1 knocked down was more than $400 \%$ of controls $\left(\bar{x}_{\text {Control }}=11821.3, \bar{x}_{N U C B 1}=47907.7\right)$. We found a similar interaction effect $(P=0.012$, Supplementary Table 6$)$ when analyzing survival after infection (Figure 4B). NUCB1 knockdown males survived slightly better than controls after infection with E. faecalis ( $77.8 \%$ controls vs. $83.3 \%$ NUCB1 survived five days post infection) but had lower survival after infection with $P$. rettgeri (83.3\% controls vs. $66.7 \%$ NUCB1 survived four days post infection). These results are qualitatively similar when analyzing using a different day after infection or a Cox Proportional Hazard framework (data not shown). In C. elegans, nucb-1 knockdown individuals survived $E$. faecalis exposure better than untreated individuals $(78.8 \%$ knockdown vs. $32.2 \%$ untreated, Figure 4 C). In contrast, Nucb-1 knockdown individuals survived $P$. rettgeri exposure worse than untreated individuals $(38.8 \%$ knockdown vs. 15 | P a g e 
$66.7 \%$ untreated). Again, the interaction between treatment (microbe exposure) and knockdown was significant $(P<0.0001$, Supplemental Table 7).

\section{Discussion}

D. melanogaster and C. elegans are arguably the two most well-studied invertebrate model systems. Interestingly, their ecologies are overlapping and C. elegans can even be phoretic on D. melanogaster (KIONTKE AND SUDHAUS 2006). Because of this shared ecology, we would expect that the microbes each encounter in nature are also intersecting and therefore that immune defense against these microbes would be under similar selective pressures in the two models. However, innate immunity in flies and worms appears quite different. Caenorhabditis lacks the canonical NF-KappaB pathways (Toll and Imd in Drosophila). We performed a first step in a systematic comparison how the two species defend themselves against pathogenic microbes using transcriptomic data and comparing exposure to Gram-positive E. faecalis to exposure to three different Gram-negative microbes. We find that lineage restricted genes are more likely to be differentially expressed between exposure types in Drosophila, but one-toone orthologs are more likely to be differentially expressed in Caenorhabditis. Further, among conserved, one-to-one orthologs, there was weak but significant correlation between Drosophila and Caenorhabditis in the $\log _{2}$ fold change between exposure to $E$. faecalis and all three Gram-negative microbes. However, we found very little evidence that similar gene ontologies are enriched when comparing $E$. faecalis infection to the 
Gram-negatives. These results suggest that while flies and worms share similar ecologies and are therefore likely exposed to similar microbes, they seem to fight infection in very distinct ways.

One obvious caveat is that our comparison is, necessarily, not perfect. Most of the Drosophila data utilized were from systemic infection involving pinprick with a septic needle whereas Caenorhabditis were fed microbes leading to primarily gut infection. Even when using Drosophila gut infection data, we are comparing whole organism (worm) to dissected guts (flies). Finally, the timeframe for infection dynamics could be very different in the two hosts and this difference could be exacerbated by the different routes of infection. We feel, however, that the results are probably not a result of these differences in approach. In both flies and worms, infections are strongly induced by the timepoints analyzed and those time points are during the critical period when infection outcomes are decided (DUNEAU et al. 2017). Instead, we think that the differences are more likely due to fundamentally different pathways governing immune defense in the two hosts.

If host responses are fundamentally different, the specific role that a few key genes (i.e. nucleobindin) play in both species is particularly interesting. If Nucb-1 is a Gram-negative specific immune protein in both worms and flies, but uses non-canonical pathways, it might suggest a new conserved immune pathways in these two disparate hosts. NUCB1 was previously implicated in Drosophila in immune defense, but not in Caenorhabditis (BERKEY et al. 2009). We found that in both organisms immune defense against the Gram-negative pathogen, P. rettgeri is significantly reduced when Nucb-1 expression is knocked down. Moreover, immune defense against $E$. faecalis seems to 17 | P a g e 
improve when Nucb-1 expression is knocked down. This motivates a deeper study of the roll of Nucb-1 in immune defense in both hosts: mechanisms of specific defense, regulation and upstream and downstream interactors.

Overall, our results represent an initial attempt to compare immune defense pathways between $C$. elegans and $D$. melanogaster. This work has identified genes that may be good candidates for underlying the similarities of immune defense in organisms that can share an ecological niche. Moreover, the pathogens we have used here represent bacteria that are, or are related to, human health hazards. Identifying mechanisms that are conserved between flies and worms may help us to better identify mechanisms that function in human immunity. In the long term, it may be possible to use the induction of these genes in people to evaluate infection and whether the host is responding in the most efficient manner. 
Table 1: Genes significantly differentially expressed in both Drosophila and Caenorhabditis.

\begin{tabular}{|c|c|c|c|c|c|c|c|c|}
\hline \multirow{3}{*}{ Pathogen } & \multicolumn{4}{|c|}{ Drosophila } & \multicolumn{4}{|c|}{ Caenorhabditis } \\
\hline & Gene & Base & $\log _{2}$ & $\mathbf{P}_{\text {adj }}$ & Gene & Base & $\log _{2}$ & $\mathbf{P}_{\mathbf{a d j}}$ \\
\hline & & Count & FC & & & Count & FC & \\
\hline \multirow[t]{10}{*}{ Pseudomonas } & $P u$ & 888.6 & 2.60 & $4.28 \mathrm{E}-30$ & cat-4 & 82.1 & -1.14 & $8.75 \mathrm{E}-05$ \\
\hline & aay & 1028.1 & 1.50 & 1.18E-05 & Y62E10A.13 & 402 & -0.35 & $1.88 \mathrm{E}-02$ \\
\hline & $D g p-1$ & 153.0 & 1.09 & $1.26 \mathrm{E}-03$ & $\operatorname{cgp}-1$ & 1687 & -0.6 & $2.11 \mathrm{E}-10$ \\
\hline & CG14210 & 17.8 & -1.81 & 3.85E-02 & Y40B1B.7 & 810.3 & -0.39 & 1.18E-03 \\
\hline & CG9643 & 66.0 & 0.98 & 2.70E-02 & F29B9.1 & 410.6 & -1.15 & $1.84 \mathrm{E}-15$ \\
\hline & P32 & 713.1 & 2.84 & $1.28 \mathrm{E}-24$ & cri-3 & 1702.3 & -0.93 & $1.47 \mathrm{E}-15$ \\
\hline & CG10103 & 149.4 & 0.89 & 8.29E-04 & istr-1 & 1181.6 & -0.29 & 4.44E-03 \\
\hline & NUCB1 & 957.5 & 0.89 & 4.02E-07 & nucb-1 & 132.5 & 1.47 & 3.71E-08 \\
\hline & Galt & 302.5 & -0.91 & 3.85E-02 & ZK1058.3 & 331.5 & -0.97 & 4.79E-10 \\
\hline & Esyt2 & 158.5 & 0.81 & $5.69 \mathrm{E}-04$ & esyt-2 & 149.2 & 1 & 2.20E-05 \\
\hline \multirow[t]{4}{*}{ Providencia } & ade2 & 149.9 & -1.03 & 2.09E-04 & pfas-1 & 61.4 & -1.34 & $1.28 \mathrm{E}-03$ \\
\hline & $P u$ & 888.6 & 2.59 & 5.36E-30 & cat-4 & 82.1 & -1.44 & 8.37E-06 \\
\hline & P32 & 713.1 & 2.36 & $1.28 \mathrm{E}-16$ & cri-3 & 1702.3 & -0.31 & $2.45 \mathrm{E}-02$ \\
\hline & CG6218 & 360.0 & 0.51 & 4.47E-02 & W06B4.2 & 372.3 & 0.39 & 2.15E-02 \\
\hline
\end{tabular}


Immune divergence in invertebrates

\section{Figures}

Figure 1: Differentially expressed genes are associated with orthology status in both Drosophila and Caenorhabditis but in opposite directions. The proportion of differentially expressed genes (FDR $=0.05)$ in A) Drosophila and B) Caenorhabditis exposed to E. faecalis vs. Escherichia, Pseudomonas and Providencia (see supplemental table S?? for strain names, and c) both Drosophila and Caenorhabditis control compared to Pseudomonas. ${ }^{*} \mathrm{P}<0.05$, ${ }^{* *} \mathrm{P}<0.001,{ }^{* * *} \mathrm{P}<0.0001$. 
bioRxiv preprint doi: https://doi.org/10.1101/2020.03.04.977595; this version posted March 5, 2020. The copyright holder for this preprint (which was not certified by peer review) is the author/funder. All rights reserved. No reuse allowed without permission.

Immune divergence in invertebrates
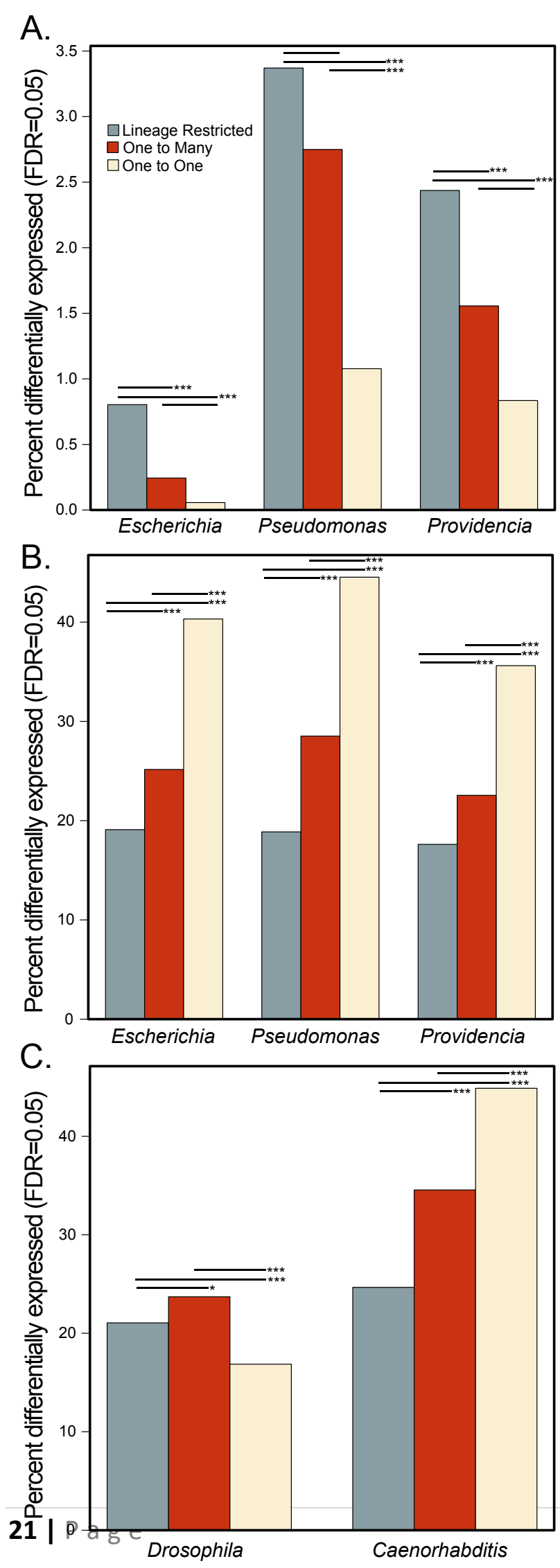
Immune divergence in invertebrates

Figure 2: Correlations between Drosophila and Caenorhabditis $\log _{2}$ fold change between E. faecalis and A) E. coli, B) Pseudomonas aeruginosa or entomophila or C) P. rettgeri. Spearman's rho value and P-values are presented in the lower right hand corner of each plot.

22 | P a g e 
bioRxiv preprint doi: https://doi.org/10.1101/2020.03.04.977595; this version posted March 5, 2020. The copyright holder for this preprint (which was not certified by peer review) is the author/funder. All rights reserved. No reuse allowed without permission.

Immune divergence in invertebrates
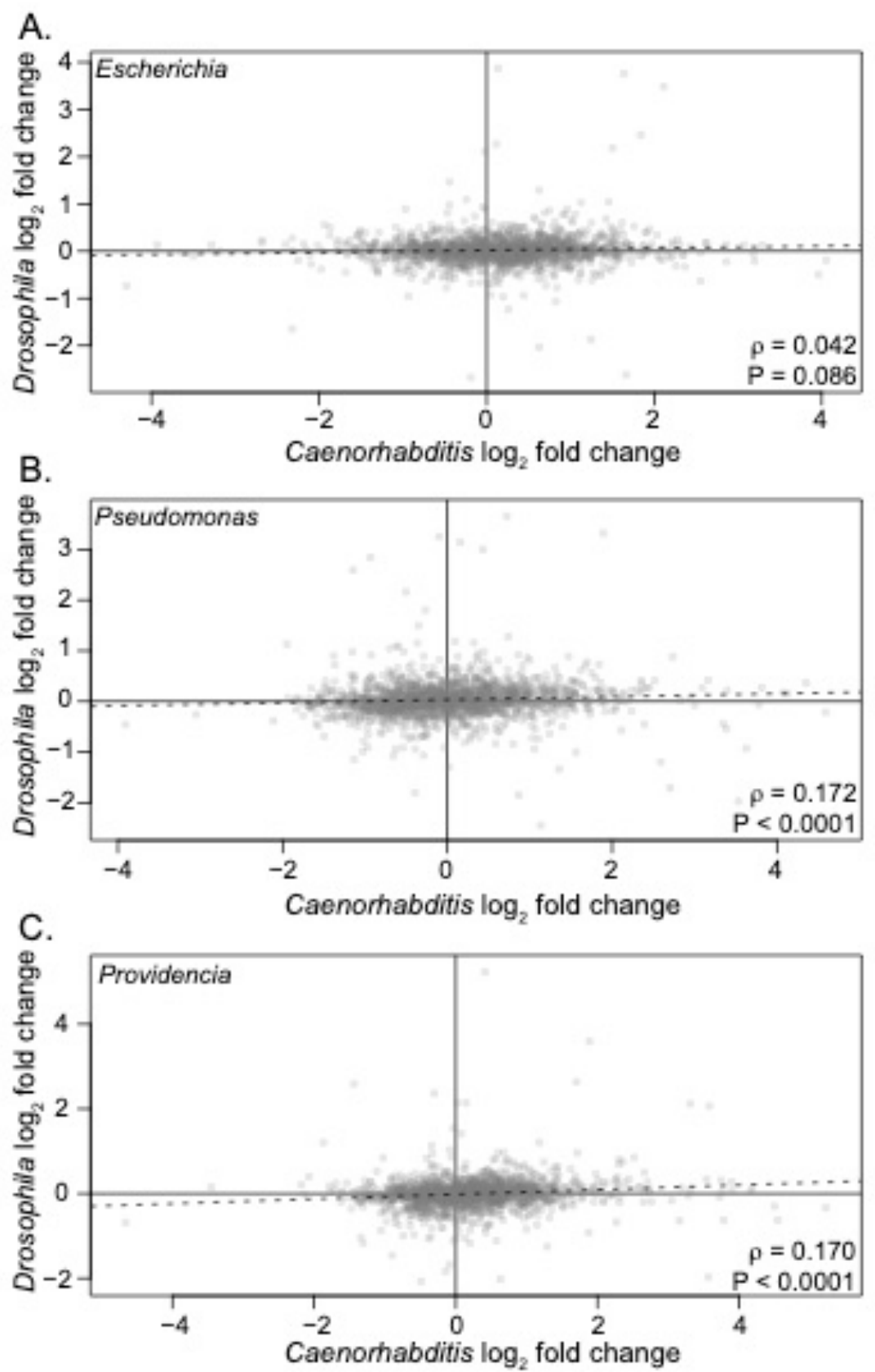

23 I P a g e 
Figure 3. Gene Ontology term enrichment overlap between comparing Grampositive E. faecalis to the three Gram-negative microbes. A) Drosophila, B) Caenorhabditis and C) all significant Drosophila Molecular Function terms to all significant Caenorhabditis Molecular Function terms.

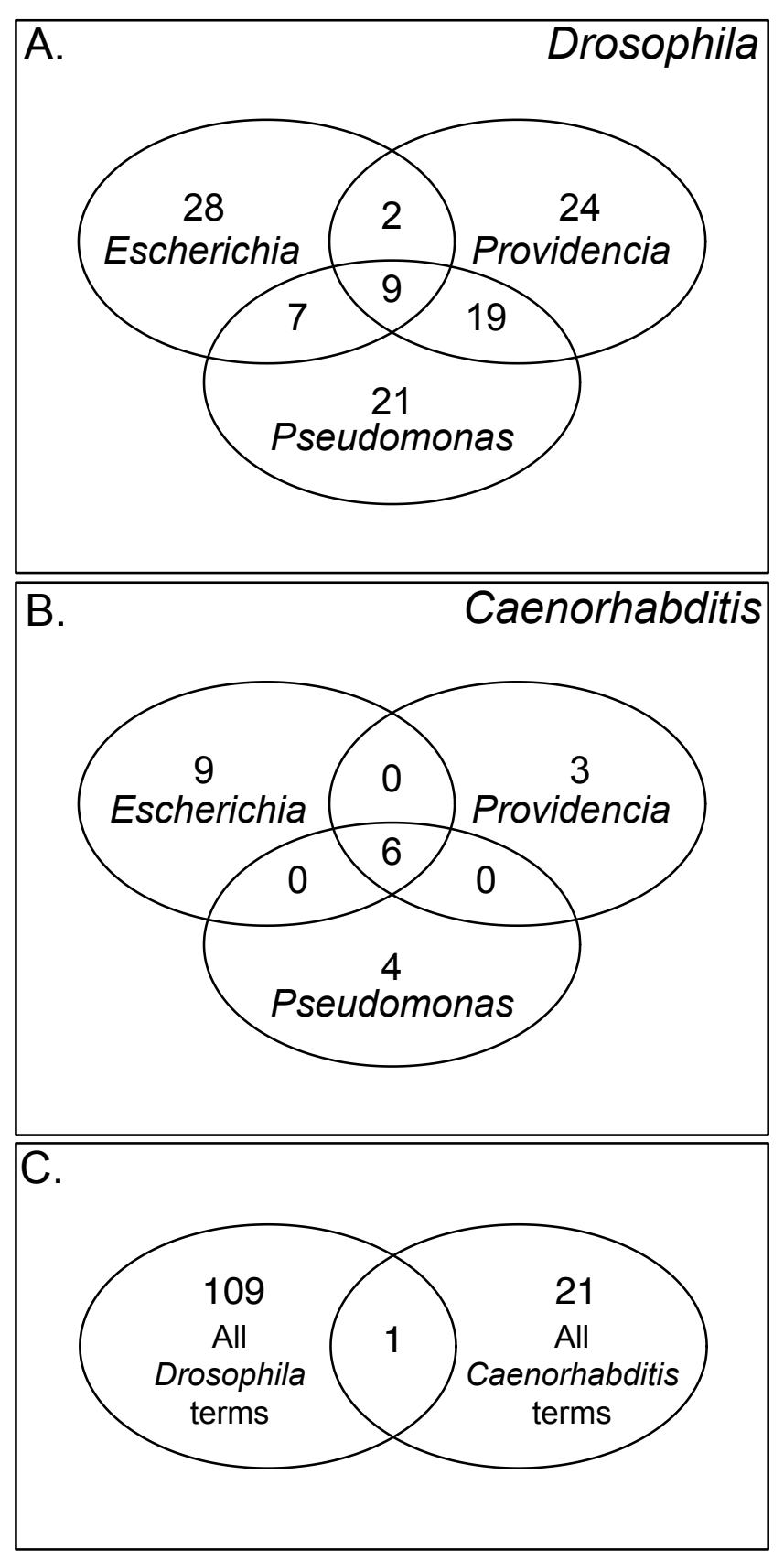


Figure 4. Nucb-1 protects specifically against Gram-negative pathogens in flies and worms. A) Bacterial load presented as natural log colony forming units (CFU) per individual fly 24 hours after infection with $E$. faecalis or $P$. rettgeri in control (fat body driver with empty cassette) or Nucb-1 knockdown (fat body driver with Nucb-1 RNAi construct). Thick bars represent means and error bars represent plus or minus one standard error of the mean. B) Survival after sterile prick or infection with E. faecalis or $P$. rettgeri in control (fat body driver with empty cassette) or Nucb-1 knockdown (fat body driver with Nucb-1 RNAi construct). $n=90$ for each sample. C. Survival after exposure to $E$. coli (standard conditions), E. faecalis or P. rettgeri in untreated or nucb-1 knockdown (worms fed E. coli expressing nucb-1 RNAi construct). $n=80-90$ for each sample. 

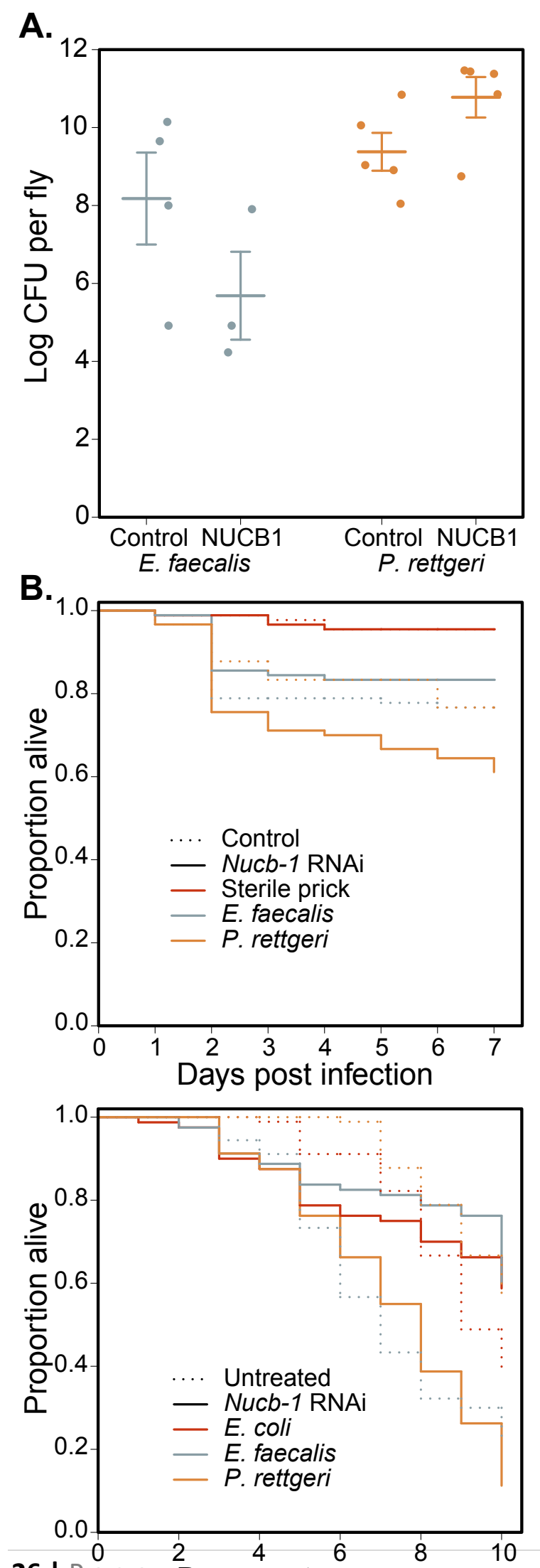

26 I P a g e Days post exposure 


\section{Supplemental Tables}

Supplemental Table 1: SRA accessions and other properties of sequencing runs for this study

\section{Supplemental Table 2: Logistic regression and Tukey HSD for orthology}

categories. LR: logistic regression, RD: residual deviance, d.f.: degrees of freedom, $\mathrm{P}(1$ :many - LinRes): Tukey HSD P-value for 1 vs. many orthologs compared to lineage restricted orthologs, etc.

Supplemental Table 3: List of all significantly enriched gene ontology (GO) terms

Supplemental Table 4. Significant overlap in enrichment of gene ontology (GO) terms

Supplemental Table 5. Drosophila bacterial load is influenced by NUCB1. Log CFU per fly analyzed by type 3 anova with genotype, treatment and their interaction as factors.

Supplemental Table 6. Drosophila survival after infection is influenced by NUCB1.

Data analyzed using logistic regression of survival five days after infection.

Supplemental Table 7. Caenorhabditis survival after infection is influenced by NUCB1. Data analyzed using logistic regression of survival five days after infection. 
Immune divergence in invertebrates

\section{Supplemental Figures}

Figure S1: Principal component plots for A) Drosophila and B) Caenorhabditis

samples. See supplemental table $\mathrm{S} 1$ and materials and methods for strain names.

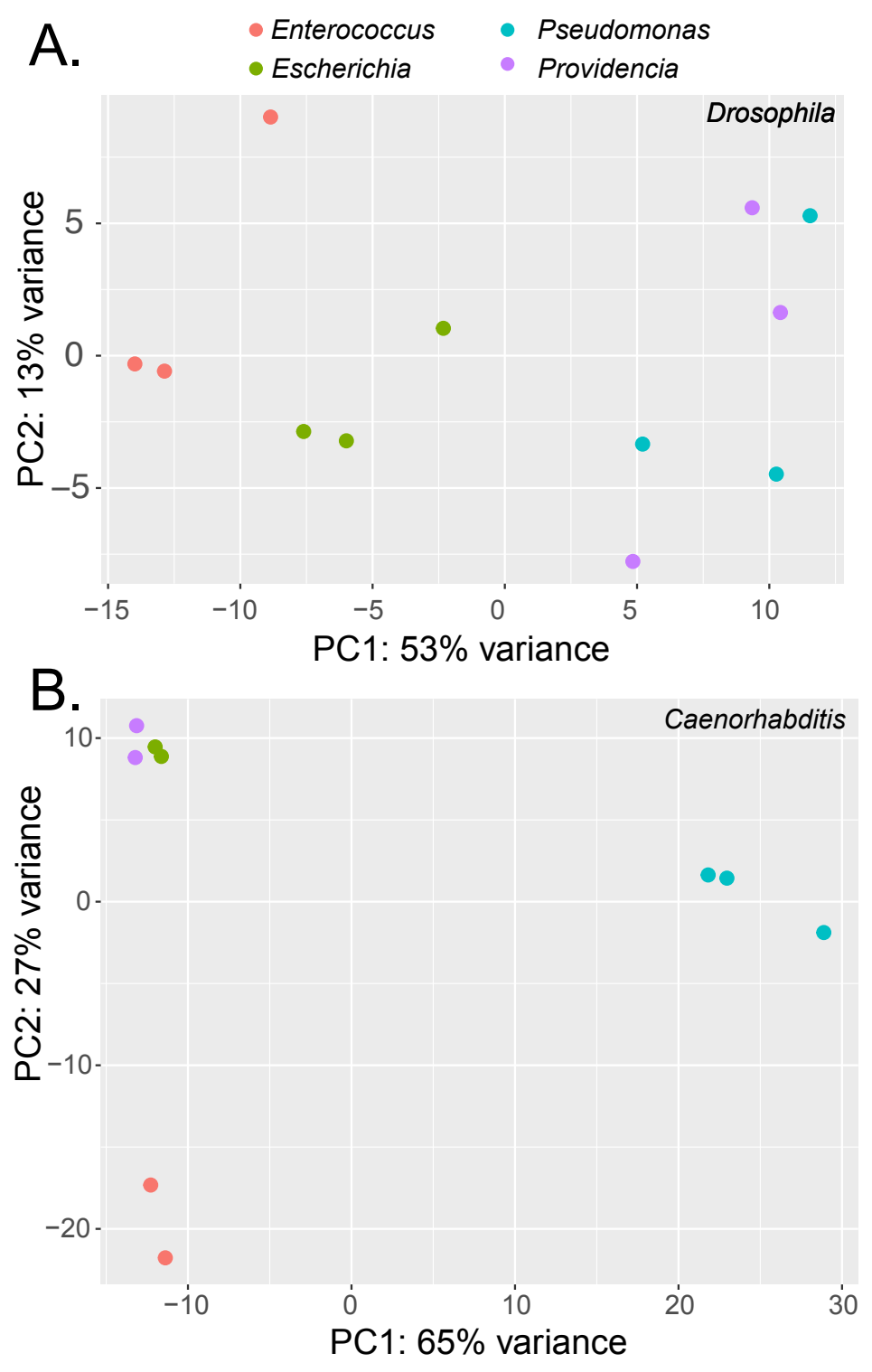

28 | P a g e 


\section{References}

Archer, H., and P. C. Phillips, 2018 Experimental evolution of independent genetic pathways for resistance to <em $>$ Pseudomonas aeruginosa $</ e m>$ pathogenicity within the nematode $<$ em $>$ Caenorhabditis remanei</em>. bioRxiv.

Berkey, C. D., N. Blow and P. I. Watnick, 2009 Genetic analysis of Drosophila melanogaster susceptibility to intestinal Vibrio cholerae infection. Cell Microbiol 11: 461-474.

Boehm, T., and J. B. Swann, 2014 Origin and evolution of adaptive immunity. Annu Rev Anim Biosci 2: 259-283.

Bou Sleiman, M., M. V. Frochaux, T. Andreani, D. Osman, R. Guigo et al., 2020 Enteric infection induces Lark-mediated intron retention at the 5 ' end of Drosophila genes. Genome Biol 21: 4.

Bray, N. L., H. Pimentel, P. Melsted and L. Pachter, 2016 Near-optimal probabilistic RNA-seq quantification. Nat Biotechnol 34: 525-527.

Brenner, S., 1974 The genetics of Caenorhabditis elegans. Genetics 77: 71-94.

Buchon, N., N. Silverman and S. Cherry, 2014 Immunity in Drosophila melanogaster-from microbial recognition to whole-organism physiology. Nat Rev Immunol 14: 796-810.

Dierking, K., W. Yang and H. Schulenburg, 2016 Antimicrobial effectors in the nematode Caenorhabditis elegans: an outgroup to the Arthropoda. Philos Trans R Soc Lond B Biol Sci 371.

Downing, T., P. Cormican, C. O'Farrelly, D. G. Bradley and A. T. Lloyd, 2009 Evidence of the adaptive evolution of immune genes in chicken. BMC Res Notes 2: 254.

Duneau, D., J. B. Ferdy, J. Revah, H. Kondolf, G. A. Ortiz et al., 2017 Stochastic variation in the initial phase of bacterial infection predicts the probability of survival in D. melanogaster. Elife 6.

Duxbury, E. M., J. P. Day, D. Maria Vespasiani, Y. Thuringer, I. Tolosana et al., 2019 Host-pathogen coevolution increases genetic variation in susceptibility to infection. Elife 8.

Ermolaeva, M. A., and B. Schumacher, 2014 Insights from the worm: the C. elegans model for innate immunity. Semin Immunol 26: 303-309. 
Hanson, M. A., B. Lemaitre and R. L. Unckless, 2019 Dynamic Evolution of Antimicrobial Peptides Underscores Trade-Offs Between Immunity and Ecological Fitness. Front Immunol 10: 2620.

Harris, T. W., V. Arnaboldi, S. Cain, J. Chan, W. J. Chen et al., 2020 WormBase: a modern Model Organism Information Resource. Nucleic Acids Res 48: D762D767.

Hill, T., B. S. Koseva and R. L. Unckless, 2019 The genome of Drosophila innubila reveals lineage-specific patterns of selection in immune genes. Mol Biol Evol.

Kao, C. Y., F. C. Los, D. L. Huffman, S. Wachi, N. Kloft et al., 2011 Global functional analyses of cellular responses to pore-forming toxins. PLoS Pathog 7: e1001314.

Kim, L. K., U. Y. Choi, H. S. Cho, J. S. Lee, W. B. Lee et al., 2007 Down-regulation of NF-kappaB target genes by the AP-1 and STAT complex during the innate immune response in Drosophila. PLoS Biol 5: e238.

Kiontke, K., and W. Sudhaus, 2006 Ecology of Caenorhabditis species. WormBook: 114.

Love, M. I., W. Huber and S. Anders, 2014 Moderated estimation of fold change and dispersion for RNA-seq data with DESeq2. Genome Biol 15: 550.

Martin, N., J. Singh and A. Aballay, 2017 Natural Genetic Variation in the Caenorhabditis elegans Response to Pseudomonas aeruginosa. G3 (Bethesda) 7: 1137-1147.

Mortimer, N. T., B. Z. Kacsoh, E. S. Keebaugh and T. A. Schlenke, 2012 Mgat1dependent $\mathrm{N}$-glycosylation of membrane components primes Drosophila melanogaster blood cells for the cellular encapsulation response. PLoS Pathog 8: e1002819.

Nielsen, R., C. Bustamante, A. G. Clark, S. Glanowski, T. B. Sackton et al., 2005 A scan for positively selected genes in the genomes of humans and chimpanzees. PLoS Biol 3: e170.

Obbard, D. J., J. J. Welch, K. W. Kim and F. M. Jiggins, 2009 Quantifying adaptive evolution in the Drosophila immune system. PLoS Genet 5: e1000698.

Pujol, N., O. Zugasti, D. Wong, C. Couillault, C. L. Kurz et al., 2008 Anti-fungal innate immunity in C. elegans is enhanced by evolutionary diversification of antimicrobial peptides. PLoS Pathog 4: e1000105.

R Development Core Team, 2015 R: A language and environment for statistical computing, pp. R Foundation for Statistical Computing, Vienna, Austria. 
Roxstrom-Lindquist, K., O. Terenius and I. Faye, 2004 Parasite-specific immune response in adult Drosophila melanogaster: a genomic study. EMBO Rep 5: 207212.

Sackton, T. B., B. P. Lazzaro, T. A. Schlenke, J. D. Evans, D. Hultmark et al., 2007 Dynamic evolution of the innate immune system in Drosophila. Nat Genet 39: 1461-1468.

Schulenburg, H., M. P. Hoeppner, J. Weiner, 3rd and E. Bornberg-Bauer, 2008 Specificity of the innate immune system and diversity of C-type lectin domain (CTLD) proteins in the nematode Caenorhabditis elegans. Immunobiology 213: 237-250.

Shultz, A. J., and T. B. Sackton, 2019 Immune genes are hotspots of shared positive selection across birds and mammals. Elife 8 .

Sim, S., and M. L. Hibberd, 2016 Caenorhabditis elegans susceptibility to gut Enterococcus faecalis infection is associated with fat metabolism and epithelial junction integrity. BMC Microbiol 16: 6 .

Sonnhammer, E. L., and G. Ostlund, 2015 InParanoid 8: orthology analysis between 273 proteomes, mostly eukaryotic. Nucleic Acids Res 43: D234-239.

Thurmond, J., J. L. Goodman, V. B. Strelets, H. Attrill, L. S. Gramates et al., 2019 FlyBase 2.0: the next generation. Nucleic Acids Res 47: D759-D765.

Troha, K., J. H. Im, J. Revah, B. P. Lazzaro and N. Buchon, 2018 Comparative transcriptomics reveals CrebA as a novel regulator of infection tolerance in $D$. melanogaster. PLoS Pathog 14: e1006847.

Vasquez-Rifo, A., I. Veksler-Lublinsky, Z. Cheng, F. M. Ausubel and V. Ambros, 2019 The Pseudomonas aeruginosa accessory genome elements influence virulence towards Caenorhabditis elegans. Genome Biol 20: 270.

Viljakainen, L., J. D. Evans, M. Hasselmann, O. Rueppell, S. Tingek et al., 2009 Rapid evolution of immune proteins in social insects. Mol Biol Evol 26: 1791-1801.

Xie, M., and R. Roy, 2012 Increased levels of hydrogen peroxide induce a HIF-1dependent modification of lipid metabolism in AMPK compromised C. elegans dauer larvae. Cell Metab 16: 322-335. 